\title{
DESCRIPTION OF FABRIC THICKNESS AND ROUGHNESS ON THE BASIS OF FABRIC STRUCTURE PARAMETERS
}

\author{
Brigita Kolcavova Sirkova
}

Technical University of Liberec, Faculty of Textile Engineering, Department of Textile Technologies, Liberec, Czech Republic Studentská 2, 46117 Liberec 1, Telephone: +420 48535 3274; Fax: +420 485353542

E-mail: brigita.kolcavova@tul.cz

\begin{abstract}
:
This paper focuses on the evaluation of thread interlacing and its influence on some of the end-use properties of woven fabric, especially its influence fabric thickness and roughness. Weaves and their interlacing structural models, different thread positions in the fabric, and thread compression in thread interlacing are important not only for final design but for final fabric properties. From the weave viewpoint, it is necessary to distinguish the specific interlacing in the longitudinal and in the transverse section as well as the dimension and design of the binding repeat.
\end{abstract}

\section{Key words:}

Thickness, woven fabric, weave, model, structure, roughness.

\section{Introduction}

Mutual interlacing of two sets of threads creates woven fabric. The manner of the mutual interlacing of threads defines the final fabric structure and mechanical and end-use properties. The shape of the binding wave and basic geometry of the binding cell changes according to the dimension and number of threads in the weave repeat or in the binding cell [1]. The whole process of weaving is the process of the formation binding point. Their dimensions and the tension gradually change from the cloth fell in the forming zone as far as the place with a steady state inside the fabric. Every irregularity in the balance of the variable forces, in the deformation of the binding point (cell), the accessibility of the sets, and in the stability of the weaving, etc., can be deduced from the description of the mutual relations between the tension and the geometrical changes of the binding cell $[2,3,4,5]$. For the weave of the fabric, it is characteristic that the pattern of the binding is repeated regularly (periodically) across the whole fabric width and it is continuous.

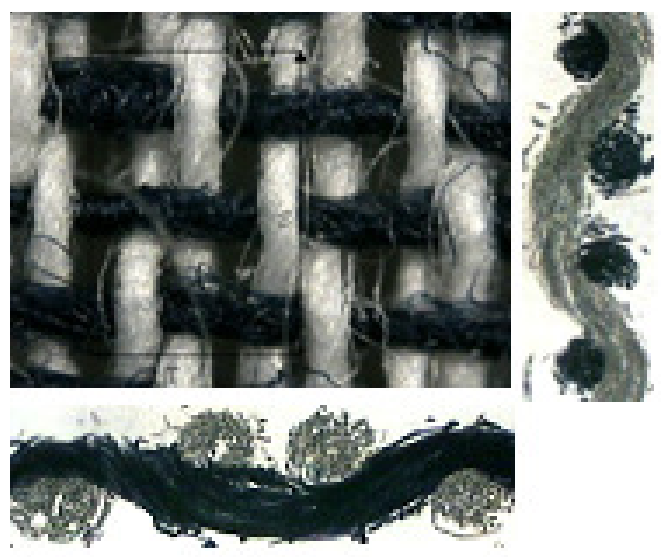

Figure 1. Depiction of real woven fabric and its longitudinal and transverse cross-section.

In the forming zone it is possible to evaluate changes in the interlacing which are caused by the difference in the warp and weft tension. In steady state, it is necessary to respect the regularity of the thread interlacing. This regularity defines the final quality of the woven fabric; cross-sections in this case have to be identical. Each irregularity or difference in the crosssection determines a fabric fault. The structure of the woven fabric is usually defined by the weave, the material of the yarn, thread density, and yarn count [4]. Fabric properties are important in each industry domain. The final fabric structure determines some fabric properties, such as fabric thickness, area covering, rubbing (fastness), pilling, air-permeability, etc. The above-mentioned properties depend on the input yarn properties as well as the input fabric properties [3, 7]. Research work has been done focusing on the weave's influence on the fabric thickness, air-permeability, area covering. Fabric thickness is dependent on the fabric weave as well as the thread's position in the binding repeat. If we have the same yarn parameters (yarn count, twist, yarn under fill, etc.), higher derived weaves with a long float have greater fabric thickness than plain weave with a small float. The same effect arises with the determination of the fabric's air-permeability. A contrary effect arises in the determination of the fabric area covering. The fabric area covering is dependent on the density of both sets of threads. If we have similar interlacing in the fabrics we can assume identical area covering for these fabrics [1].

\section{Measuring fabric thickness and fabric roughness}

Measuring the roughness of woven fabric based on the Kawabata Evaluation System (KES)

The Kawabata Evaluation System (KES) is a series of instruments used to measure those textile material properties that enable prediction of the aesthetic qualities perceived by human touch. KES instruments quantify a garment material's tactile qualities through objective measurement of the mechanical properties related to comfort perception. With low forces applied, as in manipulating / touching fabrics, the Kawabata instruments define the role played by tensile (stretch), shear stiffness (drape), bending rigidity (flexing), compression (thickness, softness), and surface friction and roughness (next to skin) on tactile sensations (see Figures 1, 


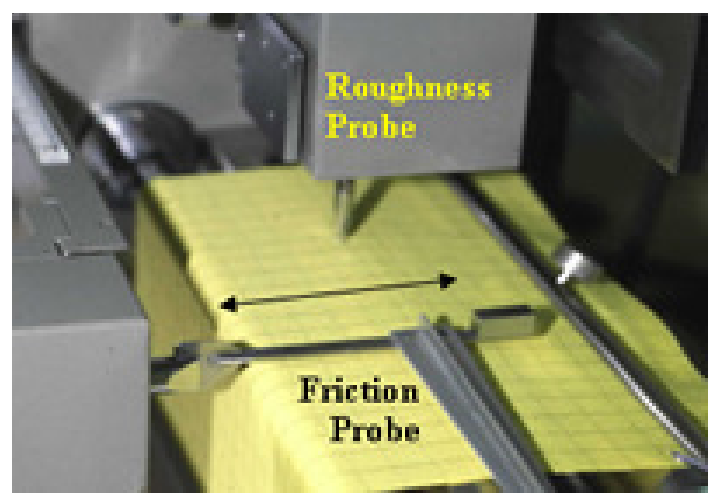

Figure 2. KES-FB4 Surface Tester.

$2,3)$. This analytical power, combined with the capability to characterize energy loss in mechanical deformation and recovery processes, provides an unparalleled tool for use in fabric hand analysis. KES provides a unique capability, not only to predict human response, but also to provide an understanding of how the variables of fibre, yarn, fabric construction and finish contribute to perceptions of comfort. The surface properties of friction (resistance / drag) and surface contour (roughness) are determined using the KES-FB4 Surface Tester (SMD - geometric roughness, micron; higher values corresponds to geometrically rougher surface) [6].

\section{Measuring the fabric thickness}

Fabric thickness is defined as perpendicular distance through the fabric, which determines the dimension between the upper and lower side of the fabric [2].
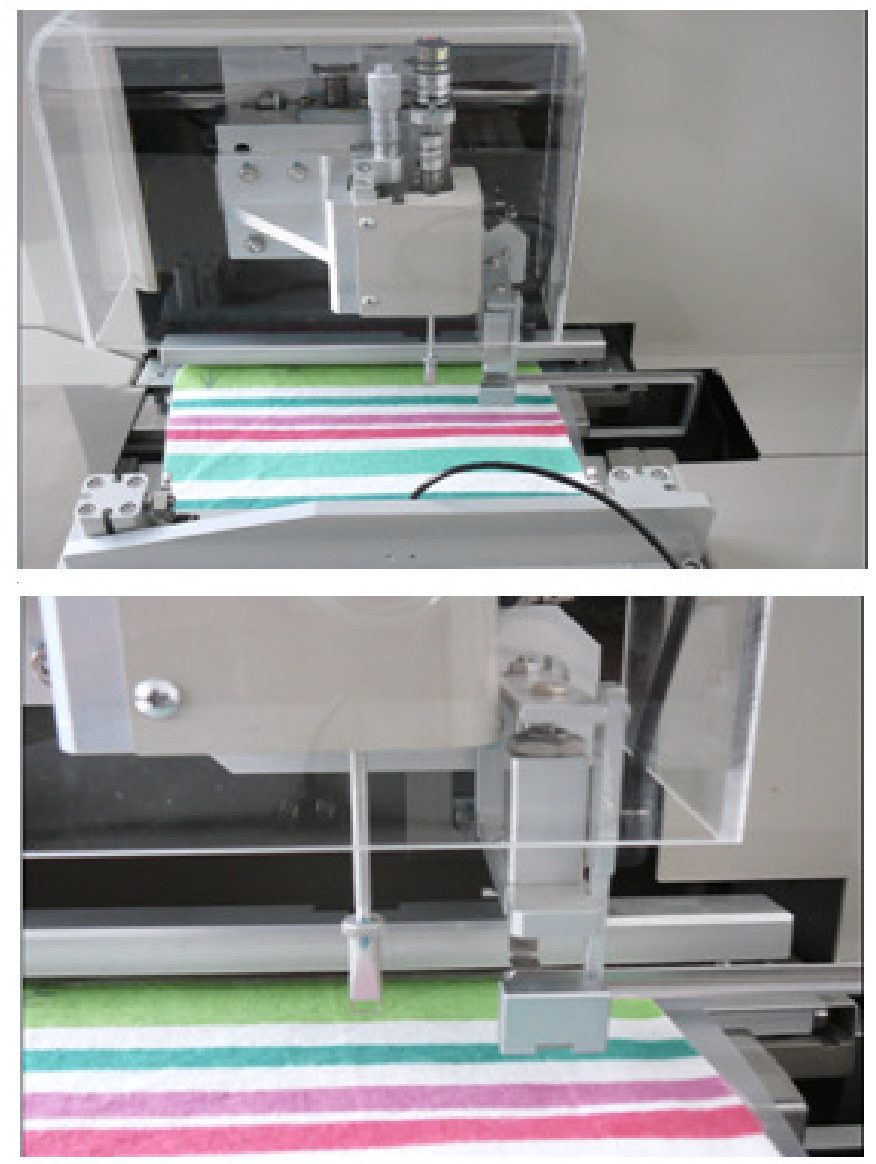

Figure 3. Surface tester for longitudinal and transverse section of woven fabric.

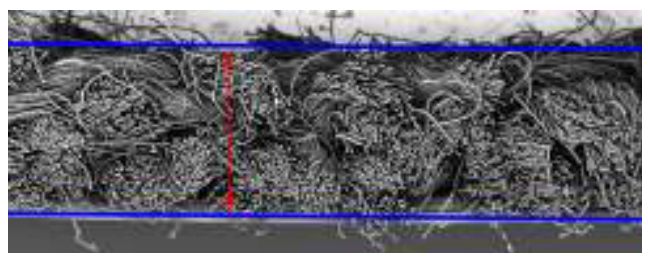

Figure 4. Longitudinal cross-section in fabric

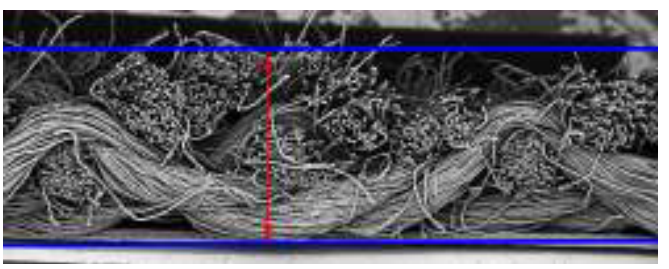

Figure 5. Transverse cross section in fabric.

According to ISO 5084, this is the vertical distance between two defined surfaces, when the fabric has $1 \mathrm{kPa}$ pressure or lower. The Kawabata Evaluation System can be used for fabric thickness determination too [6]. In this case, fabric thickness is measured by measuring compression properties: the effective dimension of the specimen is a compressed circular area of $2 \mathrm{~cm}^{2}$. The specimen is compressed by two circularplates of steel having an area $2 \mathrm{~cm}^{2}$. The velocity of the compression is $20 \mathrm{micron} / \mathrm{sec}$ and the pressure attained is $50 \mathrm{~g} / \mathrm{cm}^{2}$. The fabric thickness is taken as the thickness when $P($ pressure $)=0,5 \mathrm{gf} / \mathrm{cm}^{2}$ and the unit is taken in $T[\mathrm{~mm}]$.

\section{Geometric model for description of fabric thickness and fabric roughness}

\section{Prediction of fabric thickness}

Fabric thickness is dependent on the fabric weave as well at the thread's position in the binding repeat $[10,11]$. Yarns with identical parameters (yarn count, twist, yarn under fill, etc.) in combination with higher derived weaves with a long float have greater fabric thickness than plain weave with a small float.

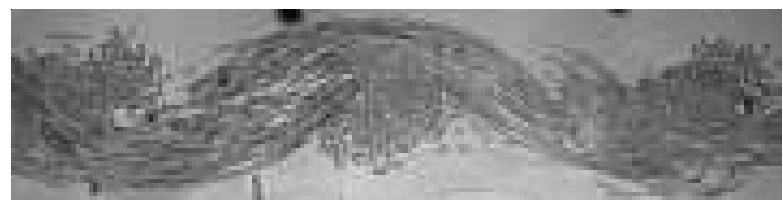

Figure 6. Longitudinal cross-section in plane fabric with weft sett=9picks $/ 100 \mathrm{~mm}$.

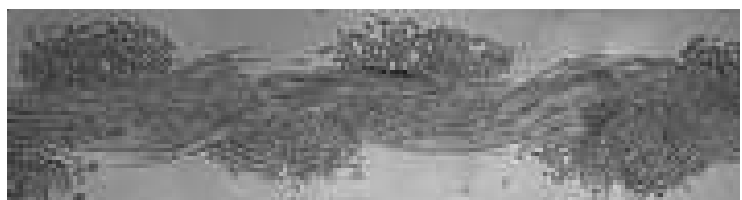

Figure 7. Transverse cross-section in plane fabric with warp sett=16 ends $/ 100 \mathrm{~mm}$.

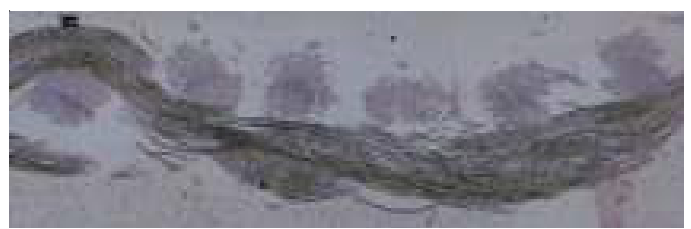

Figure 8. Longitudinal cross-section in satin fabric with weft sett=16picks $/ 100 \mathrm{~mm}$. 


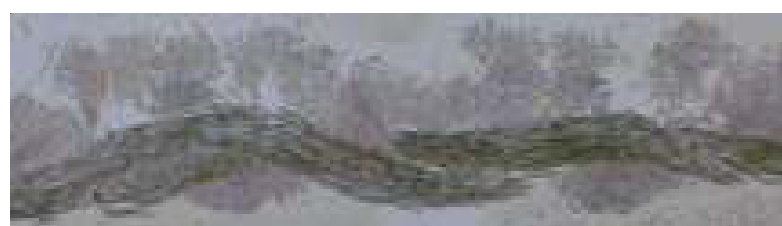

Figure 9. Transverse cross section-in satin fabric with warp sett=16 ends $/ 100 \mathrm{~mm}$.

For prediction of fabric thickness, it is possible to use the geometric model described on the basis of fabric structure and warp and weft waviness $[1,12]$. In the under-mentioned model (1) the main influence is exerted by the yarn diameter and weft and warp waviness.

thickness $[m m]=\left[\left(d_{0}+d_{u}\right)+\left[\frac{d_{0}+d_{u}}{2} \cdot e 1-\frac{d_{o}+d_{u}}{2} \cdot(1-e 1)\right] \mid\right] \cdot f^{m} \cdot \beta$

where: $d_{o, u}$ - warp and weft diameter, e1 - warp waviness, $f^{m}$ interlacing coefficient, $C$ - yarn compression in fabric (the yarn deformation in interlacing).

\section{Prediction of fabric roughness}

Roughness is a surface micro-geometry which is defined as the sum of unevenness (geometric deviations) of the surface with relatively small distances $[10,13]$. It is an important parameter influencing subjective hand feeling and connected with the behaviour of textiles layers in mutual contact. Fabric roughness depends on the fabric geometry description, thread interlacing, and yarn irregularity.

Thread interlacing is defined by the interlacing structural model. It is possible to distinguish four structural cells in the weave repeat (Figure 11).

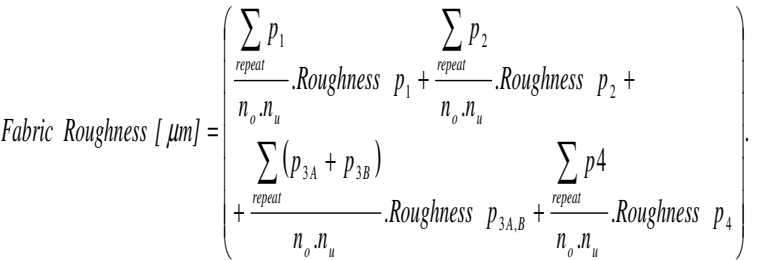

$$
\begin{aligned}
& \frac{100}{\frac{D_{0}+D_{u}}{2}} \cdot \frac{100}{100-C V} \cdot 10^{3}
\end{aligned}
$$

where: $D_{u, 0}$ threads / 100mm] - weft, warp sett, $p_{1-4}-$ pores in weave, yarn irregularity $\mathrm{CV}$.

\section{Comparison of theoretical and experimental values of fabric thickness and roughness}

Experimental data for fabric thickness was measured according to ISO 5084 and compared with values calculated according to equation (1). Roughness is determined using the KES-FB4 Surface Tester (SMD - geometric roughness, micron; higher values corresponds to geometrically rougher surfaces). In the above-mentioned graphs we can see the behaviour of fabric properties and a comparison of theoretical values with experimental values of the fabric thickness and roughness. Parameters of fabric samples were: 100\%POP yarn and $100 \%$ CO yarn in three counts 20 tex, 29,5 tex and 45 tex in both directions. All fabrics are in plain weave.
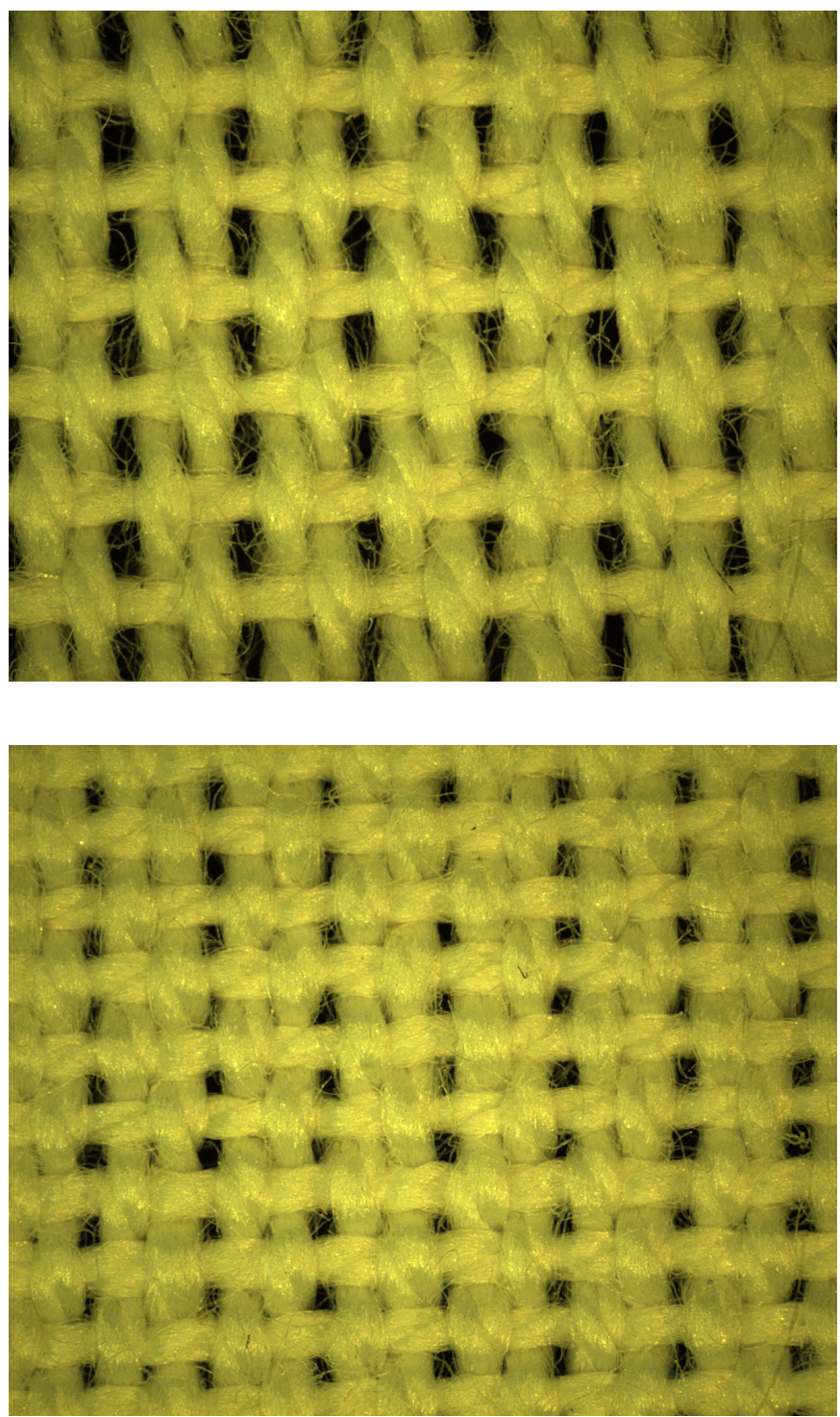

Figure 10. Illustration of weft density influence on woven fabric areal cove and roughness.

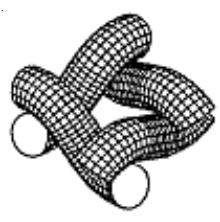

Full interlacing

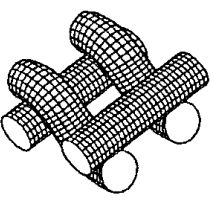

Double effect

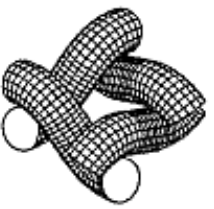

Partial interlacing

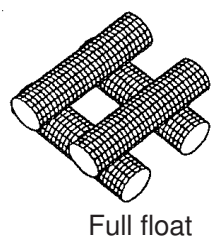

Figure 11. Interlacing structural models.

\section{Conclusion}

This paper focused on the evaluation of thread interlacing and its influence on some fabric properties. Weaves and different thread positions in the fabric are important not only for final design but for final fabric properties. Fabric thickness is dependent on the definition of the yarn diameter, yarn 


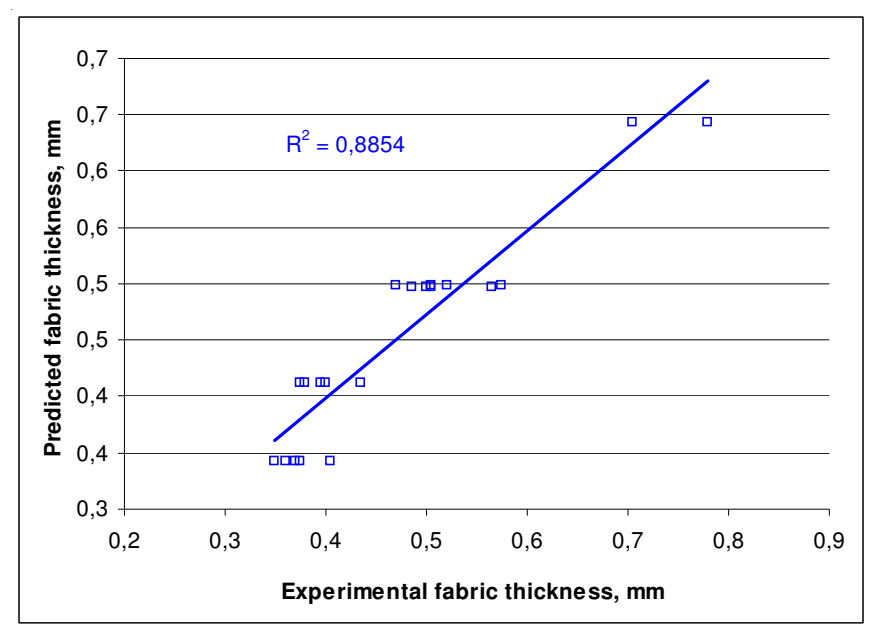

Figure 12. Comparison of experimental and predicted fabric thickness.

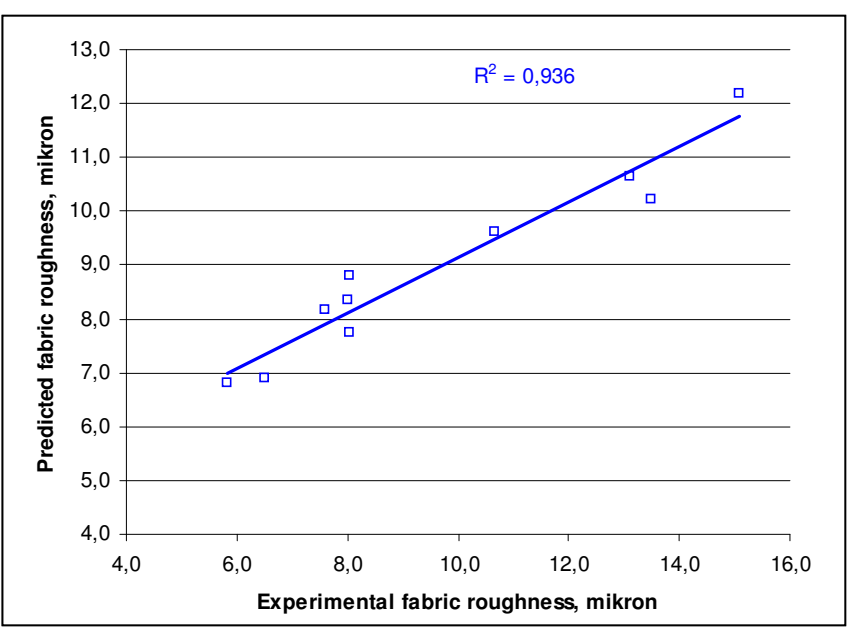

Figure 13. Comparison of experimental and predicted fabric roughness.

compression in interlacing, and on the float part in the weave repeat. Fabric roughness is dependent on the weave and the number of individual structural pores in the weave, as well as on thread densities and yarn irregularity.

\section{Acknowledgement}

This work was supported by Research Projects Textile II No. 1 M0553 and was published in the 18th Strutex conference Structure and Structural Mechanics of Textiles - held in November 2011 at the Technical University of Liberec.

\section{References:}

1. Nosek, S.: Weaving theory I, Dum techniky, Pardubice 1988.

2. Kremenakova, D., Kolcavova Sirkova, B., Mertova, I.: Internal standards, Research centre, Liberec 2004.

3. Szosland, J.: 'Kszta'3towanie wªsnoœci tkanin poprzez kszta³towanie fazy ich struktury' (in Polish, 'Designing of woven fabric features by designing the phase of their structure'), Architektura Tekstyliów, No. 1-3, 1999.

4. Wang, Y., Sun, X.: Determining the geometry of textile performs using finite element analysis, 15th Annual Technical Conference for Composites, Sept., 23-27, 2000.

5. Backer, S.: The relationship between the structural geometry of textile and its physical properties, I: Literature review. Text. Res. J. 1948, 18: 650-658.

6. Textile Protection And Comfort Center, North Carolina State University, http://www.tx.ncsu.edu/tpacc/comfortperformance/kawabata-evaluation-system.cfm.

7. Kolcavova Sirkova, B.: The influence of threads interlacing on the mechanical properties of the woven fabrics, IMCEP 2003 Innovation and modelling of clothing engineering processes, University of Maribor, Maribor, Slovenia, 2003.

8. Behera, B.K., Hari, P.K.: Woven textile structure, Theory and applications, Woodhead Publishing Limited, ISBN 978-1-84569-514-9 (book), 2010.

9. Neckar, B.: Compression and Packing Density of Fibrous Assemblies. Textile Research Journal, pp. 123-130 Vol. 67 No. 3.

10. Kremenakova D., Mertova I., Kolcavová Sirkova B.: Computer aided textile design 'LibTex', Indian Journal of Fiber \& Textile Research, India, Vol.33, December 2008, pp. 40-404, ISSN 0971-0426.

11. Milasius V.: Woven Fabric's Cross-Section: Problems, Theory, and Experimental Data, Fibres and Textiles in Eastern Europe No 4(23)/98, pp. 48-50.

12. Oloffson B.: "A general model of a fabric as a geometric mechanical structure", J. Textile Inst. Nr11, 55, pp. 541557; 1964.

13. Ozgen, B., Gong, H.: Yarn geometry in woven fabric, Textile Research Journal, May 2011; vol. 81, 7: pp. 738-745. 